\title{
ANTOCIANINAS TOTALES Y PARÁMETROS DE COLOR EN LÍNEAS DE MAÍZ MORADO
}

\section{TOTAL ANTHOCYANINS AND COLOR PARAMETERS IN PURPLE CORN INBRED LINES}

\author{
C. Gabriela Mendoza-Mendoza', Ma. del Carmen Mendoza-Castillo*, \\ Adriana Delgado-Alvarado², Fernando Castillo-González', \\ Takeo Á. Kato-Yamakake ${ }^{1}$ y Serafín Cruz-Izquierdo ${ }^{1}$
}

\begin{abstract}
'Postgrado en Recursos Genéticos y Productividad-Genética, Campus Montecillo, Colegio de Postgraduados. km 36.5 Carretera México-Texcoco. 56230, Montecillo, Texcoco, Edo. de México. Tel. 01 (595) 9520200 Ext. 1524. ${ }^{2}$ Campus Puebla, Colegio de Postgraduados. Boulevard Forjadores de Puebla No. 205. 72760, Santiago Momoxpan, Municipio San Pedro Cholula, Puebla, México. Tel. 01(222) 2851445 Ext. 2026.
\end{abstract}

*Autor para correspondencia (camen@colpos.mx)

\section{RESUMEN}

El maíz morado (Zea mays L.) contiene pigmentos naturales del tipo antocianinas con propiedades bioactivas de aprovechamiento en la industria nutracéutica, alimentaria y textil. Para considerar al cultivo del maíz como una fuente importante de este flavonoide, es necesario valorar el potencial productivo y orientar el mejoramiento genético hacia un incremento en su concentración total en grano y en otras estructuras de almacenamiento. En el programa de mejoramiento genético de maíces pigmentados del Colegio de Postgraduados, durante el ciclo agrícola primavera-verano 2015 se seleccionaron 124 líneas $\mathrm{S}_{2}$, las cuales mayoritariamente fueron clasificadas mediante una escala de color como granos de color morado intenso y morado rojizo; también se determinó el contenido de antocianinas totales (AT) en grano completo, pericarpio y aleurona y el color en la escala HunterLab, con la finalidad de valorar el potencial productivo de antocianinas, caracterizarlas por sus parámetros de color, determinar la estructura del grano con mayor AT e identificar líneas élite para su uso en el programa de mejoramiento genético. En promedio, las líneas tuvieron $0.085,0.959$ y $0.031 \mathrm{~g}$ de AT $100 \mathrm{~g}^{-1}$ en grano completo, pericarpio y aleurona, respectivamente; AT fue mayor en pericarpio que en grano y aleurona. Los valores promedio de color en los grano fueron $10.5,3.3,2.2$ y $39.9^{\circ}$ para $\mathrm{L}, \mathrm{a}$, b y ángulo hue, respectivamente, lo que indica colores de rojo a amarillo oscuro, que no fueron afines con la escala de color (azul, rojo, morado y sus variantes) pero que son parámetros cuantitativos del color. Según su croma (4.1), el color de grano fue de baja saturación (color apagado). En general, las líneas mostraron alto potencial productivo de antocianinas; no obstante, sólo $25 \%$ de ellas fueron consideradas como líneas élite, y las diez con mayor potencial productivo fueron: 118, 122, 198, $121,120,117,147,161,123$ y 124.

Palabras clave: Zea mays, antocianinas totales, líneas endogámicas, maíz morado, potencial productivo, selección.

\section{SUMMARY}

Purple corn (Zea mays L.) contains natural pigments of the anthocyanin type. These pigments have bioactive properties that may be used in the nutraceutical, food and textile industries. Before affirming that maize is an important source of this flavonoid, it is necessary to assess its productive potential and to direct plant breeding efforts towards increasing total concentration in grains and other storage structures. In the pigmented maize breeding program at Colegio de Postgraduados, during the Spring-Summer
2015 crop season, $124 \mathrm{~S}_{2}$ inbred lines were selected and most were classified on a color scale as intense purple and reddish-purple. Total anthocyanin content (TAC) in the whole grain, pericarp and aleurone, as well as color in the HunterLab scale were also determined to assess their anthocyanins productive potential, to characterize them by their color parameters, to identify the grain structure with the highest TAC, and to select elite inbred lines that might be used in the breeding program. On average, the inbred lines had $0.085,0.959$ and $0.031 \mathrm{~g}$ of TAC $100 \mathrm{~g}^{-1}$ in the whole grain, pericarp and aleurone, respectively; TAC was higher in pericarp than in the whole grain and aleurone. Mean color values in grain were $10.5,3.3,2.2$ and $39.9^{\circ}$ for $\mathrm{L}, \mathrm{a}, \mathrm{b}$ and hue angle, respectively. These values indicate red to dark yellow colors, which were not related to the color scale (blue, red, purple and their variants) nonetheless, they are quantitative parameters of color. According to their chroma value (4.1), grain color was of low saturation (dull color). In general, the lines showed high productive potential of anthocyanins; nevertheless, only $25 \%$ of them were considered as elite lines, and the ten lines with the highest productive potential were: $118,122,198,121,120,117,147,161,123$ and 124.

Index words: Zea mays, total anthocyanins, inbred lines, purple corn, productive potential, selection.

\section{INTRODUCCIÓN}

La alta incidencia de enfermedades tales como cardiovasculares, diabetes, cáncer, desnutrición, entre otras (Pandey et al., 2010; Shoji y Nakashima, 2004), así como la necesidad de encontrar productos menos contaminantes para el ambiente (Lobell et al., 2008) y la demanda de productos naturales menos procesados, con menores o nulos efectos secundarios sobre la salud (Mateus y Freitas, 2009), en conjunto con el descubrimiento de diversos compuestos fitoquímicos que tienen amplio potencial de aprovechamiento en la industria, han permitido el crecimiento y desarrollo de investigaciones en el área nutracéutica y de los alimentos funcionales (Bhowmik et al., 2013). En tales avances científicos se ha descubierto que las plantas producen antocianinas, las cuales son metabolitos 
secundarios (flavonoides) con propiedades antioxidantes que pueden incidir favorablemente sobre la salud al reducir la manifestación de enfermedades coronarias, así como por su actividad anticancerígena, efecto vasoprotector, antiinflamatorio, prevención de déficits de memoria, funciones neuroprotectoras, efectos citoprotectores, entre otros (Soto-Vaca et al., 2012); asimismo, pueden ser utilizadas como colorante natural en los alimentos y en la industria textil (Boo et al., 2012).

Dichos efectos benéficos han impulsado la exploración de especies que puedan ser utilizadas para la extracción de antocianinas, como la soya negra (Glycine max L.), papa morada (Solanum tuberosum L.), uva (Vitis vinifera L.), zarzamora(Rubus ulmifolius L.), col morada (Brassica oleracea var. capitata f. rubra), arándano(Vaccinium myrtillus L.), así como el maíz de grano de color azul, rojo, morado o negro, que puede ser una de las fuentes ideales, ya que sintetiza al pigmento en el cariópside (Yang et al., 2009); además, representa uno de los tres principales granos que alimentan a la humanidad, pues forma parte de la canasta básica en la alimentación de la población de países en vías de desarrollo, constituye la principal fuente de carbohidratos (Atlin et al., 2011), es un cereal de producción económica y fácil de almacenar que no se deteriora de forma inmediata si se mantiene seco (Escribano-Bailón et al., 2004).

No obstante, para su plena adopción como fuente de extracción de antocianinas, se requieren variedades de maíz altamente productivas, tanto de grano como de antocianinas, y en una siguiente etapa, determinar la cantidad en que se encuentran almacenadas. En las últimas décadas se han realizado diversos estudios sobre las antocianinas del maíz, algunos con la finalidad de detectar su presencia en poblaciones nativas, razas y materiales mejorados, para estimar la concentración del pigmento y para definir los tipos de antocianinas (Fernández et al., 2013; LopezMartinez et al., 2009; Salinas et al., 2012; Urias-Peraldí et al., 2013).

De dichas pesquisas se ha concluido que los maíces nativos pigmentados poseen un gran potencial de aprovechamiento, constituyen un destacado reservorio y patrimonio genético; sin embargo, tienen bajo rendimiento de grano, alta susceptibilidad a enfermedades, ciclo biológico largo, así como la complejidad del carácter de color de grano, por lo que resulta imprescindible la implementación de metodologías de mejoramiento genético para transformarlos en una fuente más redituable y eficiente para la extracción del colorante natural.

Bajo este esquema, y teniendo en cuenta que el maíz morado es rico en compuestos bioactivos (Ramos-Escudero et al., 2012), en el programa de mejoramiento genético de maíces pigmentados del Posgrado de Recursos Genéticos y Productividad-Genética del Colegio de Postgraduados, desde el año 2013 se han estudiado los maíces morados de San Juan Ixtenco, Tlaxcala (México). Paralelamente se han derivado líneas endogámicas, las cuales evidentemente (por su coloración) producen antocianinas; sin embargo se desconoce la magnitud de su potencial productivo.

Por lo anterior, los objetivos del trabajo consistieron en valorar el potencial productivo de 124 líneas $S_{2}$ a través de la determinación de la concentración de antocianinas totales en grano completo, pericarpio y capa de aleurona, además de realizar su caracterización por color de grano, e identificar y seleccionar aquéllas líneas con mayor potencial productivo de antocianinas para su conservación y utilización en el programa de mejoramiento genético.

\section{MATERIALES Y MÉTODOS}

\section{Material genético}

En el Colegio de Postgraduados (CP), Campus Montecillo (Mt), durante el ciclo primavera-verano (P-V) 2014 se establecieron plantas provenientes de 100 mazorcas de polinización fraternal, las cuales fueron formadas en el ciclo P-V 2013 en Mt y dos localidades ubicadas en San Juan Ixtenco, Tlaxcala (Tlax), a partir de 22 poblaciones nativas de maíz morado producidas en Tlax en 2012, y algunas poblaciones pigmentadas formadas en el programa de mejoramiento de Mt. Las 100 mazorcas se eligieron de un grupo de 363 provenientes de cruza fraternal, por tener color de grano morado y azul oscuro; en las plantas establecidas se realizaron autofecundaciones obteniéndose 289 líneas $S_{1}$, de las cuales 100 fueron seleccionadas por su coloración (grano azul y morado) y sanidad, y fueron establecidas en el ciclo P-V 2015 para avanzar en su nivel de endogamia a $S_{2}$.

Se obtuvieron 255 líneas $S_{2}$; sin embargo, sólo 124 (48.6 $\%$ del total de líneas $S_{2}$ ) se seleccionaron por su sanidad y por tener un color de grano considerado dentro de las cinco categorías que integraron la escala de color de grano (Figura 1). Dichas líneas fueron estudiadas en laboratorio en relación con la magnitud de su potencial productivo de antocianinas y se identificaron con la numeración del 100 al 226, con excepción de los números 196, 218 y 225. Como testigos se consideraron los materiales Az-2 (azul), B-1 (blanco) y RP-1 (rojo), aunque éstos no fueron incluidos en el análisis estadístico para evitar una sobreestimación del potencial productivo de las líneas, pues éstas mostraron un contenido de antocianinas totales superior; no obstante, los testigos se utilizaron como referencia para señalar las diferencias entre colores de grano. 


\section{Preparación de muestras, extracción y cuantificación de antocianinas totales}

Para determinar el contenido de antocianinas totales en el pericarpio (ATper) y en la capa de aleurona (ATal), de cada línea se tomaron 25 granos de la parte central de la mazorca, se sumergieron $3.5 \mathrm{~h}$ en agua destilada para disectar el pericarpio del resto del grano (una sola pieza conformada por la capa de aleurona, endospermo y embrión). Las estructuras se deshidrataron en una estufa de secado a $40{ }^{\circ} \mathrm{C}$ durante $48 \mathrm{~h}$. Consecutivamente se pesó cada estructura (Pper $=$ Peso de pericarpio y Pal $=$ Peso de aleurona, endospermo y embrión, ambos en g) y la biomasa total del grano (PTgr) se obtuvo al sumar Pper y Pal. A partir de Pper, Pal y PTgr se determinó el porcentaje de pericarpio (\% per) y el porcentaje del resto del grano (\% al) que conformaron a PTgr. Después, cada estructura fue pulverizada $(0.5 \mathrm{~mm})$; en el caso de la aleurona, dado que es un tejido muy fino y difícil de separar del resto del grano, se molió junto con el endospermo y el embrión.

La extracción se hizo por ultrasonido a partir de $25 \mathrm{mg}$ de muestra $($ Per $=$ pericarpio o Ale $=$ aleurona $)$ en tubos Eppendorf $\circledast$ de $2 \mathrm{~mL}$ se obtuvieron 3 repeticiones por muestra. A cada tubo se le agregó etanol $96 \%$ y $1.5 \mathrm{~N}$ de ácido clorhídrico $(85: 15 \mathrm{v} / \mathrm{v})$, en muestras de Ale se utilizó una proporción sólido-líquido de 1:25 y en muestras de Per de 1:80. Inmediatamente después, los tubos se colocaron en un baño de ultrasonido (Ultrasonic Cleaner AutoSCIENCE ${ }^{\circledR}$ AS-5150B, Zhejiang, China) durante 15 min, y a continuación se centrifugaron en una micro-centrífuga (HERMLE®) Z326K, Wehingen, Germany) a 11510 Xg durante 5 min; después, el sobrenadante fue colectado; se procedió a realizar una segunda extracción por el mismo procedimiento y los dos extractos fueron concentrados.

La cuantificación de antocianinas se realizó mediante un espectrofotómetro de microplaca (Thermo Scientific ${ }^{\circledR}$ Varioskan Flash, Vantaa, Finland), en el que las lecturas de absorbancia se realizaron a $535 \mathrm{~nm}$; de cada extracto se tomaron tres alícuotas de $200 \mu \mathrm{L}$. El cálculo de antocianinas totales se hizo con la fórmula: $A T=\frac{A \cdot v e x t \cdot P M \cdot F D}{\varepsilon \cdot P m u}$ donde $A T$ = contenido de antocianinas totales en $\mathrm{mg} \mathrm{g}^{-1}$ de grano para ATgr, en $\mathrm{mg} \mathrm{g}^{-1}$ de pericarpio para ATper y en $\mathrm{mg} \mathrm{g}^{-1}$ de biomasa, que es el grano sin pericarpio para ATal, $\mathrm{A}=$ absorbancia, Vext $=$ volumen de extracción en $\mathrm{mL}, \mathrm{PM}=$ peso molecular de la cianidina-3-glucósido (449.2 $\left.\mathrm{g} \mathrm{mol}^{-1}\right)$, FD = factor de dilución, $\varepsilon=$ coeficiente de extinción molar de la cianidina-3-glucósido $\left(26900 \mathrm{~L} \mathrm{~cm}^{-1} \mathrm{mg}^{-1}\right)$ y Pmu $=$ peso de la muestra en $\mathrm{g}$.

Se utilizó el PM y $\varepsilon$ de la cianidina 3-glucósido puesto que esta antocianina es la más común en las plantas. Una vez que se determinó ATper y ATal, los resultados se trans- formaron a g de antocianinas totales. El contenido de antocianinas totales en el grano (ATgr) se determinó con la fórmula AT $g r=\left(\frac{\% \text { per } \times \text { ATper }}{100}\right)+\left(\frac{\% a l \times A T a l}{100}\right)$.

\section{Clasificación de líneas, orden jerárquico y selección de líneas élite}

Con base en ATgr, ATper y ATal, y considerando una variable a la vez, las líneas se clasificaron como: a) sobresalientes, b) intermedias y c) no sobresalientes, utilizando como criterio de clasificación que $25 \%$ del total de líneas, es decir, 31 líneas tuvieran los valores de AT mayores, que fueran superiores al AT promedio y con AT igual o inferior al promedio, respectivamente. Los testigos únicamente fueron utilizados como referencia y no fueron clasificados en estos grupos. Posteriormente, para identificar la magnitud del potencial productivo de antocianinas de las líneas, así como para ubicarlas en un orden jerárquico (señalar su posición del 1 al 124, iniciando por la línea de mayor potencial productivo) se detectó la presencia o ausencia de atributos deseables, donde se consideró que la línea exhibió un atributo cuando AT fue superior a la media, pudiendo tener desde 0 (cuando ninguna de las variables tuvo AT superior a la media) hasta tres atributos (cuando ATgr, ATper y ATal fueron mayores que la media). El orden jerárquico se realizó por: número de atributos exhibidos, ATgr, ATper y ATal, acomodados del valor superior al valor inferior. Finalmente se categorizó como líneas élite a las primeras 31 líneas de la lista, que fueron equivalentes a $25 \%$ del total de líneas estudiadas.

\section{Parámetros de color. escala de color de grano y escala HunterLab}

Para caracterizar visualmente la coloración del grano se estableció convencionalmente una escala de color (Figura 1), la cual consistió en clasificar las líneas según su color de grano como: 1 = azul oscuro, 2 = rojo, 3 = morado azuloso, 4 = morado rojizo y 5 = morado intenso. Aunque los granos de las 124 líneas fueron seleccionados por su coloración oscura e intensa, dentro de este grupo de líneas hubo variación de la oscuridad de cada color, por lo que el extremo inferior de la escala (Figura 1.1) indica que los granos son más claros y azules, y el extremo superior (Figura 1.5), que los granos son morados y más oscuros. Además de ayudar a la caracterización del color de grano, la escala de color se estableció para determinar el índice de correlación existente entre el color y la concentración de antocianinas totales.

Con base en la escala HunterLab se realizó un estudio colorimétrico con el equipo HunterLab Modelo 45/O (Reston, Virginia) que generó los parámetros de color $L$, a y b; 

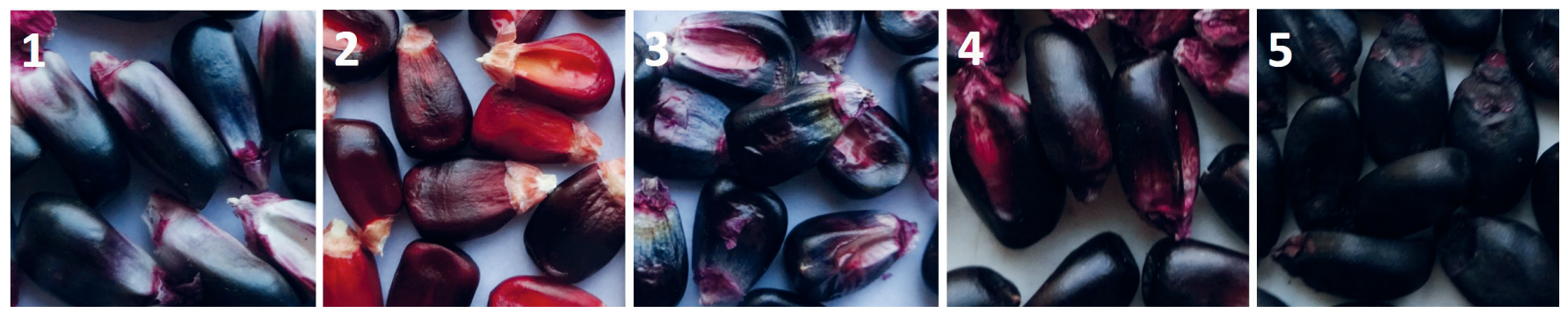

Figura 1. Escala de color de grano utilizada para evaluar visualmente el color de grano de líneas $\mathrm{S}_{2}$ de maíz morado: $1=$ azul oscuro, 2 = rojo, 3 = morado azuloso, 4 = morado rojizo y 5 = morado intenso.

se tomaron cinco lecturas en cada línea. La variable $L$ se refiere a luminosidad, que señala que el color es claro si $51 \leq L \leq 100$ y oscuro, si $0 \leq L \leq 50$. Los valores de a y $\mathrm{b}$ indican las coordenadas de cromaticidad en la escala HunterLab. Además se estimó el ángulo hue $(H)$ con la fórmula $\mathrm{H}=\tan ^{-1}(\mathrm{~b} / \mathrm{a})$ (grados); y croma, $\mathrm{C}=\sqrt{\mathrm{a}^{2}+\mathrm{b}^{2}}$.

\section{Análisis estadístico}

El análisis de los datos se hizo con el paquete estadístico SAS (Statistical Analysis System) para Windows versión 9.0; se realizó análisis de varianza con el diseño completamente al azar, así como la comparación de medias entre los tratamientos con la prueba de Tukey $(a=0.05)$.

\section{RESULTADOS Y DISCUSIÓN}

El análisis de varianza indicó que hubo diferencias altamente significativas $(P \leq 0.01)$ entre líneas en todas las variables evaluadas (Cuadro 1). En los parámetros de la escala HunterLab se encontró que los coeficientes de variación fueron altos $(27.2,48.4,25.5,35.9$ y $31.7 \%$ para $L$, a, b, C y H, respectivamente), lo cual se atribuyó a que la distribución del color en la superficie del grano no fue uniforme y a que su acomodo en la muestra fue aleatorio; sin embargo, las mediciones fueron útiles ya que describieron al color de grano cuantitativamente.

En cuanto a la escala de color de grano, ésta permitió conocer la frecuencia de cada categoría de color, donde 68 líneas tuvieron un color de grano morado intenso (Figura 1.5), 45 fueron de grano morado rojizo (Figura 1.4) y el resto fueron de grano rojo (Figura 1.2), morado azuloso (Figura 1.3) y azul oscuro (Figura 1.1), lo que en términos generales garantizó que las líneas tuvieran alto rendimiento de antocianinas, dada la correlación positiva que existe entre la intensidad del color observado con la concentración del pigmento (Espinosa et al., 2009).

\section{Valoración del potencial productivo de antocianinas}

El contenido de antocianinas totales (AT) de las 124 líneas $\mathrm{S}_{2}$ de maíz corroboró que es promisorio el potencial productivo de pigmentos, ya que tuvieron un intervalo de concentración de antocianinas en el grano (ATgr) de 0.013 a $0.309 \mathrm{~g}$ de AT $100 \mathrm{~g}^{-1}$ de grano. Al analizar el contenido de antocianinas en el pericarpio (ATper) y en la aleurona (ATal), estructuras de almacenamiento del grano, se encontró que sintetizaron y acumularon el colorante natural en ambas $y$, notablemente, en mayor cantidad en el pericarpio que en la aleurona, cuyos intervalos fueron de 0.033 a $3.761 \mathrm{~g}$ de AT $100 \mathrm{~g}^{-1}$ de pericarpio y 0.002 a $0.067 \mathrm{~g}_{\text {de AT }} 100 \mathrm{~g}^{-1} \mathrm{de}$ biomasa en la aleurona. En promedio, las líneas tuvieron $0.085,0.959,0.031 \mathrm{~g}$ de AT $100 \mathrm{~g}^{-1}$ en grano, pericarpio y aleurona, respectivamente.

La amplia magnitud de los intervalos de AT en cada estructura estudiada evidenció que las líneas poseen potencial productivo de antocianinas y que en la mayoría éste es superior al potencial de los materiales Az-2 y RP-1, que también almacenan este pigmento en la capa de aleurona y en ambas estructuras (Cuadro 2); inclusive, dicho potencial podría ser incrementado mediante la formación de híbridos pigmentados.

Cuadro 1. Cuadrados medios del análisis de varianza para el contenido de antocianinas totales y parámetros de color en líneas $\mathrm{S}_{2}$ de maíz morado.

\begin{tabular}{|c|c|c|c|c|c|c|c|c|c|c|}
\hline FV & GL & ATgr & ATper & ATal & $\mathrm{GL}$ & L & a & $\mathrm{b}$ & C & $\mathrm{H}$ \\
\hline Líneas & 123 & $0.002 * \star$ & $5.69 * *$ & $1.8 \times 10^{-3} \star *$ & 123 & $50.2 * *$ & $14.9 * *$ & $1.78 * *$ & $12.2 * *$ & $922.9 * *$ \\
\hline Error & 984 & $7.7 \times 10^{-5}$ & 0.017 & $1.0 \times 10^{-5}$ & 492 & 8.2 & 2.5 & 0.322 & 2.2 & 159.8 \\
\hline
\end{tabular}

FV: fuente de variación; GL: grados de libertad; ATgr: antocianinas totales en el grano completo; ATper: antocianinas totales en el pericarpio; ATal: antocianinas totales en la capa de aleurona; L= luminosidad; a y b: coordenadas de cromaticidad; C: croma; $\mathrm{H}$ : ángulo hue; $* *: \mathrm{P} \leq 0.01$. 


\section{Antocianinas totales en el grano}

Al comparar el contenido de antocianinas totales en grano (ATgr) de las líneas evaluadas en este trabajo con las descritas en la literatura, además de coincidir con que el ATgr reportado en maíz varía de acuerdo con las técnicas de extracción y del germoplasma utilizado (Salinas et al., 2013), se encontró que la clasificación del color de grano es variable entre los reportes; por ejemplo, Salinas et al. (2012) indican que los granos que estudiaron fueron de color azul/morado, mientras que, de acuerdo con la escala propuesta en este trabajo, los granos son de color azul oscuro y distaron del color morado. En consecuencia, al consultar las fuentes de información para cotejar el potencial de estas líneas fue necesario analizar el origen geográfico, fotografías del grano exhibidas en los artículos y las unidades en las que se mostraron los resultados. En la exploración se encontró que el maíz morado AREQ516540TL tuvo ATgr $=0.851 \mathrm{~g}$ de AT $100 \mathrm{~g}^{-1}$ de grano (Lopez-Martinez et al., 2009), que resultó 2.75 veces superior a la línea 118 de la colección aquí estudiada, que tuvo la concentración máxima de ATgr, y también fue similar a la concentración encontrada por Zhao et al. (2008) en un híbrido chino de grano negro (0.304 g de AT $100 \mathrm{~g}^{-1}$ de grano). Las líneas $118,122,198,121,120,212,117,129,148$ y 127, ordenadas de mayor a menor ATgr, tuvieron entre 0.309 y $0.184 \mathrm{~g}$ de AT $100 \mathrm{~g}^{-1}$ de grano. También fueron las 10 líneas con los valores máximos de ATgr, e inclusive más productivas que una variedad comercial de maíz morado de grano ceroso, cuyo rendimiento fue de $0.165 \mathrm{~g}$ de AT $100 \mathrm{~g}^{-1}$ de grano (Khampas et al., 2013).

Las líneas sobresalientes para ATgr ( $25 \%$ del total de líneas con mayor AT), que incluyeron a las 10 mencionadas previamente, así como a las líneas 147, 161, 158, 176, 132, $123,155,154,135,124,159,144,139,167,165,173,105$, 104, 162, 149 y 103, también ordenadas de mayor a menor ATgr, tuvieron el intervalo de concentración de 0.309 a $0.104 \mathrm{~g}$ de AT $100 \mathrm{~g}^{-1}$ de grano, las cuales con excepción de la línea 103, superaron en rendimiento al híbrido morado estudiado por Wang y Zeng (2009) que tuvo $0.106 \mathrm{~g}$ de AT $100 \mathrm{~g}^{-1}$ de grano. Además, las concentraciones fueron similares a la reportada por Espinosa et al. (2009) en granos de color púrpura (0.127 g de AT $100 \mathrm{~g}^{-1}$ de grano), así como a las de Lopez-Martinez et al. (2009) en el genotipo Mm04c1 de grano negro (0.121 g de AT $100 \mathrm{~g}^{-1}$ de grano) y a los de grano morado rojizo de la raza Arrocillo, analizados por Salinas et al. (2005) con $0.115 \mathrm{~g}$ de AT $100 \mathrm{~g}^{-1} \mathrm{de}$ grano.

En las 15 líneas $\mathrm{S}_{2}$ intermedias (AT superior a la media, 0.085 a 0.101 g de AT $100 \mathrm{~g}^{-1}$ de grano): 142, 207, 106, 136, 134, 146, 116, 114, 115, 125, 151, 214, 164, 141 y 133, así como 12 líneas del grupo de líneas no sobresalientes (AT igual o inferior a la media, 0.076 a $0.085 \mathrm{~g}$ de AT $100 \mathrm{~g}^{-1}$ de grano): 185, 137, 128, 156, 101, 171, 119, 163, 110, 178, 112 y 169, se observó que mostraron mayor concentración que la reportada por Salinas et al. (2005) para granos de la raza Peruano (0.076 g de AT $100 \mathrm{~g}^{-1}$ de grano), raza que en investigaciones relacionadas, con frecuencia es señalada como la fuente de antocianinas de maíz con mayores concentraciones (Collison et al., 2015; Ryu et al., 2013; Zhao et al., 2009; Žilić et al., 2012).

Del mismo modo, las líneas 200, 211, 145, 143, 109, 130, 140, 157 y 180 (no sobresalientes) tuvieron concentraciones de antocianinas mayores que el maíz morado boliviano Kulli (0.071 g de AT $100 \mathrm{~g}^{-1}$ de grano) descrito por Cuevas et al. (2011). Implícitamente, otras líneas del mismo grupo mostraron un desempeño superior al del cultivar chino Zihei de grano morado, cuya capacidad de acumulación de antocianinas fue de $0.055 \mathrm{~g}$ de AT $100 \mathrm{~g}^{-1}$ de grano (Yang y Zhai, 2010). Inclusive, las 10 líneas con ATgr inferiores (de 0.013 a $0.030 \mathrm{~g}$ de AT $100 \mathrm{~g}^{-1}$ de grano) tuvieron mayor potencial de sintetizar y acumular antocianinas que los genotipos de referencia Az-2 y RP-1 (Cuadro 2).

Estos resultados fueron trascendentes, pues al tratarse de líneas con $75 \%$ de homocigosis y AT por encima de las reportadas para híbridos y variedades comerciales de maíz morado, se espera que para su aprovechamiento en futuras combinaciones de cruzamientos híbridos, la capacidad de producir antocianinas se incremente por efecto de heterosis (Pfeiffer y McClafferty, 2007). Además, es posible que el potencial productivo de pigmentos de las líneas pueda ser mayor, ya que en la cuantificación de antocianinas de este estudio solamente se realizaron dos extracciones consecutivas por cada muestra y el solvente de extracción utilizado fue etanol acidificado; al respecto, Yang et al. (2009) indican que con dos extracciones se obtiene 94.3 $\%$ del total de las antocianinas contenidas en la muestra y Xu et al. (2010) señalan que la extracción de este pigmento con metanol es $20 \%$ más efectiva que con etanol.

\section{Antocianinas totales en el pericarpio}

Aun cuando las líneas en estudio fueron seleccionadas por su color oscuro e intenso (Figura 1), y tratando de asegurar elevadas concentraciones de pigmento, se observó que algunas tuvieron concentraciones bajas, como las líneas 222, 205, 210, 204 con ATgr de 0.013 a $0.023 \mathrm{~g}$ de AT. Hubo una diferencia de $0.295 \mathrm{~g}$ de AT entre el valor mínimo $(0.013 \mathrm{~g}$ de AT) y el valor máximo (0.309 g de AT) de ATgr; lo cual se atribuyó a que el patrón de acumulación de antocianinas tanto en el pericarpio como en la capa de aleurona fue variable. Por ejemplo, la línea 103 pigmentó toda la superficie de las estructuras, mientras que algunas otras no colorearon todo el pericarpio o toda la superficie 
Cuadro 2. Orden jerárquico, contenido de antocianinas totales en grano, pericarpio, aleurona y parámetros de color de 20 líneas $\mathrm{S}_{2}$ de maíz morado.

\begin{tabular}{|c|c|c|c|c|c|c|c|c|c|c|}
\hline $\mathrm{O}^{(-)}$ & Línea & ATgr & ATper & ATal & $\mathrm{C}^{1}$ & L & a & $b$ & $C$ & $\mathrm{H}$ \\
\hline $1^{\text {(a) }}$ & 118 & $0.3090^{(1)}$ & $3.7609^{(1)}$ & $0.0532^{(9)}$ & 5 & 5.9 & 1.5 & 2.7 & 3.2 & 61.3 \\
\hline $2^{(a)}$ & 122 & $0.2526^{(2)}$ & $2.4035^{(9)}$ & $0.0605^{(3)}$ & 5 & 8.5 & 0.5 & 1.7 & 1.8 & 73.5 \\
\hline $3^{\text {(a) }}$ & 198 & $0.2401^{(3)}$ & $3.0417^{(4)}$ & $0.0550^{(5)}$ & 5 & 7.0 & 1.0 & 2.2 & 2.5 & 66.7 \\
\hline $4^{\text {(a) }}$ & 121 & $0.2332^{(4)}$ & $2.1627^{(12)}$ & $0.0330(57)$ & 5 & 7.1 & 1.3 & 2.2 & 2.6 & 59.2 \\
\hline $5^{\text {(a) }}$ & 120 & $0.2161^{(5)}$ & $2.6328^{(7)}$ & $0.0619^{(2)}$ & 5 & 13.2 & 2.0 & 1.5 & 2.5 & 41.2 \\
\hline $6^{\text {(a) }}$ & 117 & $0.1983^{(7)}$ & $2.6825^{(6)}$ & $0.0510^{(13)}$ & 5 & 8.4 & 1.6 & 1.9 & 2.5 & 51.8 \\
\hline 7 (a) & 147 & 0.1730 & $1.8778^{(17)}$ & $0.0447^{(26)}$ & 5 & 10.3 & 1.3 & 1.5 & 2.0 & 51.5 \\
\hline $8^{(a)}$ & 161 & $0.1646^{(12)}$ & $1.7707^{(19)}$ & $0.0493^{(15)}$ & 5 & 8.3 & 1.8 & 2.1 & 2.9 & 52.2 \\
\hline 9 (a) & 123 & $0.1315^{(16)}$ & $1.9648(15)$ & $0.0350^{(52)}$ & 5 & 7.5 & 2.3 & 2.2 & 3.2 & 45.4 \\
\hline $10^{\text {(a) }}$ & 124 & $0.1205^{(20)}$ & $1.6794^{(22)}$ & $0.0385^{(40)}$ & 5 & 7.6 & 1.3 & 1.9 & 2.4 & 56.0 \\
\hline $17^{(a)}$ & 207 & 0.0986 (33) & $0.9803^{(48)}$ & $0.0332^{(56)}$ & 5 & 8.2 & 1.5 & 1.9 & 2.5 & 55.6 \\
\hline $18^{(a)}$ & 116 & 0.0926 (38) & 1.0554 (43) & $0.0333^{(55)}$ & 5 & 10.1 & 2.2 & 1.7 & 2.8 & 36.5 \\
\hline 19 (a) & 164 & $0.0867^{(44)}$ & $1.0135^{(45)}$ & 0.0359 (45) & 4 & 9.5 & 6.0 & 3.1 & 6.8 & 27.7 \\
\hline 31 (a) & 136 & $0.0943^{(35)}$ & 1.1520 (38) & $0.0280(77)$ & 5 & 9.6 & 1.9 & 1.7 & 2.6 & 43.0 \\
\hline $34^{(b)}$ & 114 & $0.0925^{(39)}$ & 0.6910 (70) & $0.0397^{(34)}$ & 5 & 8.5 & 2.2 & 1.9 & 3.1 & 42.7 \\
\hline 92 (c) & 174 & $0.0324^{(111)}$ & $0.0517^{(118)}$ & $0.0315^{(62)}$ & 3 & 13.8 & 3.6 & 1.9 & 4.1 & 30.2 \\
\hline 100 (c) & 202 & $0.0577^{(86)}$ & 0.6089 (76) & $0.0305^{(67)}$ & 4 & 18.8 & 5.7 & 1.9 & 6.2 & 26.8 \\
\hline $122^{\text {(c) }}$ & 197 & $0.0300^{(115)}$ & $0.0330^{(124)}$ & $0.0298^{(3)}$ & 1 & 19.9 & 5.3 & 2.1 & 5.9 & 25.5 \\
\hline $123^{(c)}$ & 205 & $0.0191^{(123)}$ & $0.0419^{(120)}$ & $0.0174^{(52)}$ & 2 & 12.3 & 6.8 & 3.8 & 7.8 & 29.6 \\
\hline $124^{\text {(c) }}$ & 222 & $0.0135^{(124)}$ & $0.0710^{(115)}$ & $0.0104^{(112)}$ & 2 & 16.7 & 10.2 & 5.8 & 11.8 & 31.0 \\
\hline \multirow[t]{3}{*}{ Testigos } & $A z-2$ & 0.0258 & 0.0032 & 0.0268 & & 19.9 & 3.1 & 6.1 & 6.8 & 63 \\
\hline & B-1 & 0.00042 & 0.0007 & 0.0004 & & 53.5 & -0.4 & 14.2 & 14 & 91.6 \\
\hline & RP-1 & 0.0045 & 0.0277 & 0.0028 & & 22.6 & 10.6 & 10.5 & 15 & 44.7 \\
\hline DSH & & 0.0183 & 0.2724 & 0.0066 & & 8.0 & 4.4 & 1.6 & 4.1 & 35.4 \\
\hline Media & & 0.0853 & 0.9597 & 0.0315 & & 10.5 & 3.3 & 2.2 & 4.1 & 39.9 \\
\hline Mín & & 0.0135 & 0.0330 & 0.0020 & & 5.7 & 0.5 & 1.1 & 1.8 & 10.2 \\
\hline Máx & & 0.3090 & 3.7609 & 0.0677 & & 24.5 & 10.5 & 5.8 & 11.8 & 73.5 \\
\hline
\end{tabular}

$\mathrm{O}$ (-): Orden jerárquico de la línea acorde con su potencial productivo de antocianinas, que se encuentra entre 1 y 124 e inicia por la línea de mayor potencial productivo; letras entre paréntesis: (a) línea élite, (b) línea sobresaliente y ${ }^{(c)}$ línea no sobresaliente; ATgr: antocianinas totales en grano ( $\mathrm{g}$ de AT $100 \mathrm{~g}^{-1}$ de grano); ATper: antocianinas totales en el pericarpio ( $\mathrm{g}$ de AT $100 \mathrm{~g}^{-1}$ de pericarpio); ATal: antocianinas totales en la capa de aleurona (g de AT $100 \mathrm{~g}^{-1}$ de biomasa). En Atgr, ATper y ATal el número entre paréntesis muestra la posición que ocupó la línea en dicha variable, la cual se encuentra entre 1 y 124; $C^{1}$ es el color de grano, 1: azul oscuro, 2: rojo, 3: morado azuloso, 4: morado rojizo y 5: morado intenso; L: luminosidad; a y b: coordenadas de cromaticidad; C: croma; H: ángulo hue; DSH: diferencia significativa honesta; Mín: valor mínimo; Máx: valor máximo.

del endospermo. En algunos genotipos la porción de pericarpio cercana al pedicelo del grano no tuvo coloración (línea 107) o mostraron patrones rayados (líneas 126 y 221), mientras que en la aleurona las disparidades que se observaron fueron por diferentes tonalidades del color en granos de un mismo genotipo (línea 126), ausencia o ligera presencia del pigmento (línea 214) o presencia de moteados (línea 212).

El pericarpio acumuló 30.46 veces más AT de lo que en promedio se concentró en la aleurona; sin embargo, solamente constituyó $5.5 \%$ del peso total del grano, lo que implica que para obtener $0.959 \mathrm{~g}$ de AT (valor promedio de ATper) se necesita $1.818 \mathrm{~kg}$ de grano para extraer las antocianinas de $100 \mathrm{~g}$ de pericarpio; no obstante, si el pericarpio posee un tipo de antocianina útil para la industria nutracéutica, esta estructura es una excelente fuente de extracción de antocianinas. Así, con una producción de 3 t ha $^{-1}$ de alguna población generada con estas líneas se obtendría $1.583 \mathrm{~kg}$ de AT ha-1 ${ }^{-1}$ exclusivamente de pericarpio. 
La ATper en las líneas sobresalientes (25\% del total de líneas con mayor AT): 118, 158, 129, 198, 127, 117, 120, $212,122,132,159,121,135,173,123,148,147,167,161$, 176, 157, 124, 155, 165, 154, 214, 213, 128, 149, 110 у 143, ordenadas de mayor a menor ATper, osciló entre $3.761 \mathrm{y}$ $1.328 \mathrm{~g}$ de AT $100 \mathrm{~g}^{-1}$ de pericarpio, de las cuales sólo las últimas cinco tuvieron concentración menor a la reportada por Salinas et al. (2005) para el pericarpio de granos de maíz de las razas Arrocillo y Peruano (1.473 y $1.524 \mathrm{~g}$ de AT $100 \mathrm{~g}^{-1}$ de pericarpio, respectivamente); sin embargo, estas líneas sobresalientes acumularon más pigmento que el maíz morado de Perú que tuvo $1.06 \mathrm{~g}$ de AT $100 \mathrm{~g}^{-1}$ de pericarpio (Monroy et al., 2016). Las líneas intermedias (AT superior a la media): 144, 211, 105, 139, 151, 156, 136, 137, 199, 104, 103, 116, 130, 164, 200, 133, 207 y 171 tuvieron entre 1.320 y $0.978 \mathrm{~g}$ de AT $100 \mathrm{~g}^{-1}$ de pericarpio; las líneas 222, 186, 195, 174, 108, 205, 168, 221, 203 y 197 del grupo de líneas no sobresalientes (AT igual o inferior a la media) rindieron de 0.033 a $0.710 \mathrm{~g}$ de AT $100 \mathrm{~g}^{-1}$ de pericarpio. Todas las líneas fueron más productivas en ATper que Az-2, RP-1 y B-1 (Cuadro 2).

\section{Antocianinas totales en la aleurona}

Las líneas sobresalientes en ATal tuvieron desde 0.042 hasta $0.067 \mathrm{~g}$ de AT $100 \mathrm{~g}^{-1}$ de biomasa, y fueron: 115, 120, 122, 179, 198, 125, 146, 162, 118, 104, 108, 112, 117, 185, $161,131,103,180,203,209,172,220,219,100,105,147$, 142, 106, 144, 155 y 186, mencionadas de mayor a menor ATal. Estas líneas sintetizaron menos antocianinas que el híbrido de maíz azul élite 503 × 67 con 0.105 g de AT 100 $\mathrm{g}^{-1}$ de grano (Urias-Lugo et al., 2015) y algunos de los 25 híbridos azules evaluados por Urias-Peraldí et al. (2013), como $33 \times 58$ que produjo $0.078 \mathrm{~g}$ de AT $100 \mathrm{~g}^{-1}$ de grano. Las 10 líneas con contenido máximo en este tejido acumularon mayor cantidad de antocianinas que el maíz boliviano Tuimuru de color azul claro, cuya concentración fue de $0.051 \mathrm{~g}$ de AT $100 \mathrm{~g}^{-1}$ de grano (Cuevas et al., 2011).

En las 30 líneas intermedias (AT superior a la media) ATal fluctuó entre 0.032 y $0.041 \mathrm{~g}$ de AT $100 \mathrm{~g}^{-1}$ de biomasa, concentraciones que fueron similares $(0.030 \mathrm{~g}$ de AT 100 $\mathrm{g}^{-1}$ de grano) a las descritas para materiales de grano azul (del Pozo-Insfran et al., 2006). Asimismo, las concentraciones fueron comparables con la obtenida en el testigo Az-2 (0.027 g de AT $100 \mathrm{~g}^{-1}$ de grano). Con estos resultados se confirma que los maíces morados poseen mayor capacidad de síntesis y almacenamiento de antocianinas que los maíces azules que sólo producen el pigmento en la capa de aleurona; inclusive se encontraron líneas que, aunque no son altamente productivas en el pericarpio, con la cantidad que acumulan en la aleurona siguen siendo más productivos que los maíces azules.

\section{Líneas élite: por alto contenido de antocianinas en grano, pericarpio y aleurona}

La valoración integral del potencial productivo de las líneas $\mathrm{S}_{2}$ mostró que 74.2 \% de las 124 líneas tuvieron entre uno y tres de los atributos estudiados. Diecinueve líneas tuvieron los tres atributos, 28 líneas tuvieron dos y 45 líneas únicamente mostraron uno. Las 31 líneas que se clasificaron como élite tuvieron entre dos y tres atributos, éstas fueron: 118, 122, 198, 121, 117, 147, 161, 123, 124 144, 139, 165, 105, 104, 103, 207, 116, 164, 212, 148, 176, $132,135,167,173,162,149,142,106$ y 136, todas de grano morado intenso (Figura 1.5), con excepción de las líneas 164, 167 y 149, cuyo color fue morado rojizo (Figura 1.4). En el Cuadro 2 se muestra el orden que tomaron las líneas; es decir, su posición en función del potencial productivo de antocianinas que presentaron, el contenido de pigmento en el grano completo, pericarpio, aleurona y los parámetros de color de 20 de las 124 líneas $S_{2}$ estudiadas. Destacaron las líneas 118, 122, 198 y 120, pues en ATgr, ATper y ATal se ubicaron en las primeras cinco posiciones por su contenido sobresaliente de AT. En contraste, las líneas 174, 202, 197, 205 y 222 (no sobresalientes) son líneas que se eliminarán del programa de mejoramiento dado su limitada capacidad de acumular antocianinas.

\section{Parámetros de color en la escala HunterLab}

Las mediciones de L oscilaron entre 5.7 y 24.5, en promedio $L=10.5$ (Cuadro 2); es decir, el color de los granos fue oscuro, muy cercano al negro. Un total de 108 líneas fueron estadísticamente iguales, con intervalo de 5.7 a 13.7 y en su mayoría se trató de granos de color morado intenso (67 líneas, Figura 1.5) y morado rojizo (39 líneas, Figura 1.4), los cuales fueron más oscuros que los maíces estudiados por Salinas et al. (2005) ( $L=11.16$ a 16.08). Con respecto al parámetro a, todos los valores fueron positivos y se encontraron en el intervalo de 0.5 a 10.5 , en promedio $a=3.3$; también en $b$, todos los valores fueron positivos y se encontraron entre 1.1 y 5.8 , en promedio $b=2.2$. Dichas coordenadas se ubicaron en el eje $\mathrm{a}^{+} \mathrm{y} \mathrm{b}^{+}$; es decir, en el cuadrante de los colores rojos a amarillos.

Los valores de croma (C) fluctuaron entre 1.8 y 11.8 , en promedio $\mathrm{C}=4.1$ (Cuadro 2), en donde 94 líneas fueron estadísticamente iguales (68 de grano morado intenso, 22 de grano morado rojizo y 4 de grano morado azuloso, Figuras 1.5, 1.4 y 1.3, respectivamente), cuyo C fluctuó entre 1.8 y 4.9, lo que indica que el color de los granos de estas líneas fue de baja saturación, baja intensidad e impuro (se mezcló con una reducida cantidad de blanco), por lo que se les catalogó como de un color apagado, neutro o débil. Las 10 líneas con los valores inferiores de $C$, que se encontraron entre 1.8 y 2.5, fueron las de color de 
grano morado intenso y dentro de éstas fueron las de los granos más oscuros (pigmento en toda la superficie del grano) y tuvieron valores de $\mathrm{C}$ similares a los reportados por Salinas et al. (2005) para granos de la raza Arrocillo (C $=2.0)$, Cónico $(C=2.3)$ y Purépecha $(C=2.8)$. En contraste, las 10 líneas con $C$ máximos $(C=6.2$ a 11.8) fueron de granos morado rojizo, rojo y azul oscuro (Figuras 1.4, 1.2 y 1.1, respectivamente), cuyo color fue más "encendido" por encontrarse más saturado.

El intervalo de $\mathrm{H}$ fue de 10.2 a $73.5^{\circ}$ y en promedio $\mathrm{H}=$ $39.9^{\circ}$ (Cuadro 2), lo que significó que el tono del grano de las líneas fue del rojo intenso al anaranjado. Los valores de $\mathrm{H}$ fueron superiores (con excepción de la línea 200 con H = $10.2^{\circ}$ ) a los de las razas Peruano, Arrocillo, Cónico y Purépecha, con ángulos en el tono rojo intenso de 11.8, 10.3, 8.2 y $15.4^{\circ}$, respectivamente (Salinas et al., 2005).

Las 10 líneas con los mayores ángulos $\left(H=59.7\right.$ a $\left.73.5^{\circ}\right)$ tuvieron color de grano morado intenso (Figura 1.5), mientras que las 10 líneas con los menores ángulos $(H=10.2$ a $24.3^{\circ}$ ) fueron de color morado rojizo, morado azuloso y rojo (Figuras 1.4, 1.3 y 1.2, respectivamente). Un total de 61 líneas fueron estadísticamente similares y abarcaron $\mathrm{H}=38.1^{\circ}$ a $73.5^{\circ}$. El valor de $\mathrm{H}$ no reflejó los matices de color morado o púrpura de los granos evaluados, sino coloraciones rojizas a anaranjadas. Aunque $\mathrm{H}$ no representó el color observado y clasificado en esta investigación se detectó que el color de grano fue más oscuro conforme $\mathrm{H}$ se incrementó.

\section{CONCLUSIONES}

Las 124 líneas $S_{2}$ de maíz morado mostraron alto potencial para producir antocianinas, con rendimiento promedio de $0.085,0.959$ y $0.031 \mathrm{~g}$ de AT $100 \mathrm{~g}^{-1}$ de grano completo, de pericarpio y de aleurona, respectivamente, siendo notablemente superior en el pericarpio. Del total de líneas evaluadas, $74.2 \%$ tuvo concentraciones sobresalientes desde una hasta en las tres estructuras estudiadas. En grano la concentración fue de 0.013 a $0.309 \mathrm{~g}$ de AT $100 \mathrm{~g}^{-1} \mathrm{de}$ grano, en pericarpio de 0.033 a $3.761 \mathrm{~g}$ de AT $100 \mathrm{~g}^{-1} \mathrm{de}$

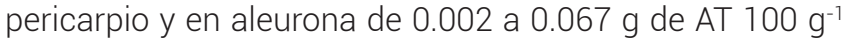
de biomasa en la aleurona. En general, el color de los granos de las líneas se caracterizó en la escala de color visual como morado intenso y, con base en los parámetros de la escala HunterLab como colores del rojo al amarillo, de baja saturación, baja intensidad, apagados y oscuros. De todas las líneas evaluadas, sólo 31 se identificaron como líneas élite, ya que expresaron el máximo potencial productivo de antocianinas, que aunado a sus características agronómicas y de rendimiento de grano favorables serán conservadas dentro del programa de mejoramiento genético de maíces pigmentados, como las líneas 118, 122, 198 y
120, que se ubicaron en las primeras posiciones por su alto contenido sobresaliente de AT en grano (de 0.216 a 0.309 $\mathrm{g}$ de AT $100 \mathrm{~g}^{-1}$ de grano), pericarpio (de 2.4035 a $3.7609 \mathrm{~g}$ de AT $100 \mathrm{~g}^{-1}$ de pericarpio) y aleurona (de 0.053 a $0.062 \mathrm{~g}$ de AT $100 \mathrm{~g}^{-1}$ de biomasa en aleurona).

\section{BIBLIOGRAFÍA}

Atlin G. N., N. Palacios, R. Babu, B. Das, S. Twumasi-Afriyie, D. K. Friesen, H. De Groote, B. Vivek and K. V. Pixley (2011) Quality protein maize: progress and prospects. Plant Breeding Reviews 34:83-131.

Bhowmik D., H. Gopinath, B. P. Kumar, S. Duraivel and K. P. S. Kumar (2013) Nutraceutical - A bright scope and opportunity of Indian healthcare market. The Pharma Innovation 1:29-41.

Boo H. O., S. J. Hwang, C. S. Bae, S. H. Park, B. G. Heo and S. Gorinstein (2012) Extraction and characterization of some natural plant pigments. Industrial Crops and Products 40:129-135.

Collison A., L. Yang, L. Dykes, S. Murray and J. M. Awika (2015) Influence of genetic background on anthocyanin and copigment composition and behavior during thermoalkaline processing of maize. Journal of Agricultural and Food Chemistry 63:5528-5538.

Cuevas M. E., S. Hillebrand, A. Antezana and P. Winterhalter (2011) Soluble and bound phenolic compounds in different Bolivian purple corn (Zea mays L.) cultivars. Journal of Agricultural and Food Chemistry 59:7068-7074.

Del Pozo-Insfran D., C. H. Brenes, S. O. Serna S. and S. T. Talcott (2006) Polyphenolic and antioxidant content of white and blue corn (Zea mays L.) products. Food Research International 39:696-703.

Escribano-Bailón M. T., C. Santos-Buelga and J. C. Rivas-Gonzalo (2004) Anthocyanins in cereals. Journal of Chromatography A 1054:129141

Espinosa T. E., M. C. Mendoza C., F. Castillo G., J. Ortiz C., A. Delgado A. y A. Carrillo S. (2009) Acumulación de antocianinas en pericarpio y aleurona del grano y sus efectos genéticos en poblaciones criollas de maíz pigmentado. Revista Fitotecnia Mexicana 32:303-309.

Fernández S. R., L. A. Morales C. y A. Gálvez M. (2013) Importancia de los maíces nativos de México en la dieta nacional. Una revisión indispensable. Revista Fitotecnia Mexicana 36:275-283.

Khampas S., K. Lertrat, K. Lomthaisong and B. Suriharn (2013) Variability in phytochemicals and antioxidant activity in corn at immaturity and physiological maturity stages. International Food Research Journal 20:3149-3157.

Lobell D. B., M. B. Burke, C. Tebaldi, M. D. Mastrandrea, W. P. Falcon and R. L. Naylor (2008) Prioritizing climate change adaptation needs for food security in 2030. Science 319:607-610.

Lopez-Martinez L. X., R. M. Oliart-Ros, G. Valerio-Alfaro, C. H. Lee, K. L. Parkin and H. S. Garcia (2009) Antioxidant activity, phenolic compounds and anthocyanins content of eighteen strains of Mexican maize. LWT - Food Science and Technology 42:1187-1192.

Mateus N. and V. de Freitas (2009) Anthocyanins as food colorants. In: Anthocyanins: Biosynthesis, Functions, and Applications. K. Gould, K. Davies and C. Winefield (eds.). Springer. New York. pp:283-304.

Monroy Y. M., R. A. F. Rodrigues, A. Sartoratto and F. A. Cabral (2016) Extraction of bioactive compounds from cob and pericarp of purple corn (Zea mays L.) by sequential extraction in fixed bed extractor using supercritical $\mathrm{CO}_{2}$, ethanol, and water as solvents. The Journal of Supercritical Fluids 107:250-259.

Pandey M., R. K. Verma and S. A. Saraf (2010) Nutraceuticals: new era of medicine and health. Asian Journal of Pharmaceutical and Clinical Research 3:11-15.

Pfeiffer W. H. and B. McClafferty (2007) HarvestPlus: breeding crops for better nutrition. Crop Science 47:S88-S105.

Ramos-Escudero F., A. M. Muñoz, C. Alvarado-Ortiz, Á. Alvarado and J. A. Yáñez (2012) Purple corn (Zea mays L.) phenolic compounds profile and its assessment as an agent against oxidative stress in isolated mouse organs. Journal of Medicinal Food 15:206215.

Ryu S. H., L. Werth, S. Nelson, J. C. Scheerens and R. C. Pratt (2013) Variation of kernel anthocyanin and carotenoid pigment content in 
USA/Mexico borderland land races of maize. Economic Botany 67:98-109.

Salinas M. Y., C. García S., B. Coutiño E. y V. A. Vidal M. (2013) Variabilidad en contenido y tipos de antocianinas en granos de color azul/ morado de poblaciones mexicanas de maíz. Revista Fitotecnia Mexicana 36:285-294.

Salinas M. Y., F. J. Cruz C., S. A. Díaz O. y F. Castillo G. (2012) Granos de maíces pigmentados de Chiapas, características físicas, contenido de antocianinas y valor nutracéutico. Revista Fitotecnia Mexicana 35:33-41.

Salinas M. Y., G. Salas S., D. Rubio H. and N. Ramos L. (2005) Characterization of anthocyanin extracts from maize kernels. Journal of Chromatographic Science 43:483-487.

Shoji Y. and H. Nakashima (2004) Nutraceutics and delivery systems. Journal of Drug Targeting 12:385-391.

Soto-Vaca A., A. Gutiérrez, J. N. Losso, Z. Xu and J. W. Finley (2012) Evolution of phenolic compounds from color and flavor problems to health benefits. Journal of Agricultural and Food Chemistry 60:6658-6677.

Urias-Lugo D. A., J. B. Heredia, S. O. Serna-Saldivar, M. D. Muy-Rangel and J. B. Valdez-Torres (2015) Total phenolics, total anthocyanins and antioxidant capacity of native and elite blue maize hybrids (Zea mays L.). CyTA- Journal of Food 13:336-339.

Urias-Peraldí M., J. A. Gutiérrez-Uribe, R. E. Preciado-Ortiz, A. S. Cruz-Morales, S. O. Serna-Saldívar and S. García-Lara (2013) Nutraceutical profiles of improved blue maize (Zea mays) hybrids for subtropical regions. Field Crops Research 141:69-76.
Wang Y. and M. Zeng (2009) Studies on Zhuo-Zi No. 1, a purple hybrid in maize (Zea mays L.). Maize Genetics Cooperation Newsletter 83:2-3.

Xu Z., J. Wu, Y. Zhang, X. Hu, X. Liao and Z. Wang (2010) Extraction of anthocyanins from red cabbage using high pressure $\mathrm{CO}_{2}$. Bioresource Technology 101:7151-7157.

Yang Z. and W. Zhai (2010) Identification and antioxidant activity of anthocyanins extracted from the seed and cob of purple corn (Zea mays L.). Innovative Food Science and Emerging Technologies 11:169-176.

Yang Z., Z. Chen, S. Yuan, W. Zhai, X. Piao and X. Piao (2009) Extraction and identification of anthocyanin from purple corn (Zea mays L.). International Journal of Food Science and Technology 44:2485-2492.

Zhao X., C. Zhang, C. Guigas, Y. Ma, M. Corrales, B. Tauscher and X. Hu (2009) Composition, antimicrobial activity and antiproliferative capacity of anthocyanin extracts of purple corn (Zea mays L.) from China. European Food Research and Technology 228:759-765.

Zhao X., M. Corrales, C. Zhang, X. Hu, Y. Ma and B. Tauscher (2008) Composition and thermal stability of anthocyanins from Chinese purple corn (Zea mays L.). Journal of Agricultural and Food Chemistry 56:10761-10766.

Žilić S., A. Serpen, G. Akıllıoğlu, V. Gökmen and J. Vančetović (2012) Phenolic compounds, carotenoids, anthocyanins, and antioxidant capacity of colored maize (Zea mays L.) kernels. Journal of Agricultural and Food Chemistry 60:1224-1231. 
